Abtheilung für Haut- und Geschlechts-Zrankheiten von Dr. med. A. Ilsenberg am Israelitischen Zrankenhause in Warschau.

\title{
Sarcomata idiopathica multiplicia pigmentosa cutis (Kaposi).
}

Von

\author{
Dr. Robert Bernard.
}

Seit der Zeit, als im Jahre $1872 \mathrm{Kaposi}^{\mathbf{1}}$ ) seine ersten Beobachtungen betreffs der idiopathischen multiplen Pigmentsarcome der Haut veröffentlichte, gaben dieselben einen Anlass zu einer ganzen Reihe ron Arbeiten, welche diesem Gegenstande gewidmet waren. Aus denselben stellte es sich heraus, dass das klinische Bild der Sarcomatosis cutis nicht immer gleich ist, so dass man einige klinische Formen dieses Leidens abzusondern vermag, wie es ja $\mathrm{K}$ aposi beispielsweise in seinem Lehrbuche darstellt. ${ }^{9}$ ) - Gleichzeitig trugen diese Arbeiten wesentlich viel dazu bei, um den histologischen Bau dieser Art von Sarcomen aufzuklären. Sie zeigten, dass Rund-, Spindelund Riesenzellensarcome (letztere sehr selten) vorkommen; auch pigmentirt oder unpigmentirt, gelagert im Derma (z. B. Typus Kaposi) oder im Hypoderma (Typus Perrin), oder auch gleichzeitig dermal und hypodermal (Typus Neumann). Es wurden auch combinirte Sarcome beschrieben: fibrosarcoma fusocellulare ( $\operatorname{Unn}$ a), angio et angiofibrosarcoma fusocellulare (Piffard). Eine rein anatomische Classification führt Unna ${ }^{3}$ ) auf: 1. Sarcoma multiplex cutaneum durum album (Typus Unna), 2. Sarcoma multiplex cutaneum durum pigmentosum

1) M. Kaposi. Idiopath. maltipl. Pigmentsarcom d. Hant. Archiv f. Dermat. a. Syph. 1872 .

$\left.{ }^{2}\right)$ Kaposi. Patholog. u. Therap. d. Hautkr. 1893.

3) Orth. Lehrbuch der spec. path. Anat. VIII. Lief. 1894. 
(Typus Piffard), 3. Sarcoma multiplex cutaneum molle (Typus Neumann), 4. Sarcoma multiplex cutaneum gummatodes (Typus Funk-Hyde), 5. Typus Perrin, 6. Acrosarcoma multiplex cutaneum telangiectodes (Typus Hebra-Kaposi).

Obwohl der histologische Bau dieser Sarcome hinreichend bearbeitet zu sein scheint, kann man dies hinsichtlich ihrer Genese nicht sagen: sie bleibt bis jetzt unaufgeklärt.

Manche, in Gemässheit der Theorie von B a b ès, nehmen die Blutgefässe als Ausgangspunkt an. Laut dieser Theorie entsteht das Sarcomgewebe in Folge abnormer Wucherung der Gefäss-Bildungskeine (les élements vaso-formateurs), welche jedoch niemals voll ausgebildete Gefässe präsentiren, sondern sie bleiben nur. mehr oder weniger Zusammenhange mit den schon präexistirenden; oder auch es entstehen die Zellen dieses Gewebes in Folge Wucherung des endothelium, adrentitia und perithelium der Blutgefässe. Von den Veränderungen der Blutgefässe erwähnt zwar fast jeder Autor, aber auch hier sind die Meinungen nicht übereinstimmend. Indem manche $\left.(\text { Perrin })^{1}\right)$ die Neubildung der Gefässe als eine primäre Erscheinung betrachten, fassen sie andere (de Amicis, Armanni) als eine secundäre auf; der Meinung dieser letzteren Autoren nach findet dieselbe erst nach der Pigmentbildung statt: "un phénomène posterieur au dépot du pigment sanguin “.2) Unna sah bei dem Typus des spindelzellensarcoms in der Uebergangszone, wo der Tumor wächst, nur Hyperämie der grösseren Gefässe und Capillaren, aber keine Neubildungen derselben; seiner Ansicht nach muss also bei diesem Typus ein "primäres angiomatöses Stadium “ (B a b̀̀ s) ausgeschlossen werden.

Hinsichtlich der Abkunft der Sarcomzellen von Gefassperithelium und endothelium ist Unna speciell in Betreff des Spindelzellentypus der Meinung, dass die Ableitung aller Zellen nur von perithelium, oder nur endothelium, abgelehnt werden muss. Zwar „der erste Ansatz der Geschwulstbildung findet sich hier, wie bei fast allen progressiven Veränderungen der Haut, allerdings an den Perithelien der Capillaren und Ueber-

1) L. Perrin. De la Sarcomatose cutanée. Thèse. Paris 1886, p. 58.

2) Perrin. 1. e. p. 58. 
Sarcomata idiopathica multiplicia pigmentosa cutis (Kaposi). 209

gangsgefässe, aber später betheiligt sich daran der gesammte zellige Antheil des Cutisgewebes".")

Folgende Meinungen der Autoren betreffen hauptsächlich das Rundzellensarcom. Was dasselbe anbetrifft, so möchte ich hier die Aufmerksamkeit auf Folgendes hinleiten: gewöhnlich nimmt man an, dass Rundzellensarcome öfters zu beobachten sind als die spindelzelligen; dies können die Beobachtungen unserer Abtheilung nicht bestätigen.

Auf der Abtheilung für Haut- und venerische Krankheiten Elsenberg's kommen mindestens seit 12 Jahren alljährlich zwei bis drei Fälle ron idiopathischen multiplen Hautsarcomen vor; jeder dieser Fälle wird unbedingt mikroskopisch untersucht und immer mit gleichem Ergebniss: ein Spindelzellensarcom. ${ }^{2}$ )

Was also die rundzelligen Sarcome anbetrifft, so nehmen manche dieselben als Lymphosarcome an, umsomehr als letztere, wie es ja bekannt ist, sich hochgradig involviren können, sei es sua sponte, oder nach medicamentösen Massnahmen. ${ }^{3}$ ) Gleichzeitig wurde die Aufmerksamkeit auf den Zusammenhang des in Rede stehenden Leidens mit Leukämie und Pseudoleukämie geleitet. In einer Reihe von Fällen (M. Joseph, Touton, Fröhlich) ${ }^{4}$ ) wurde in der That gleichzeitig Sarcomatosis cutis und Leukämie oder Pseudolenkämie beobachtet. Daraus aber kann man ja noch nicht schliessen, dass diese Hauttumoren leukämischer Abkunft wären, da doch beide Leiden gleichzeitig ein und dasselbe Individuum befallen können, ohne im causalen Zusammenhange zu stehen, umsomehr als in anderen Fällen (z. B. Kaposi) weder Blutveränderungen, noch Lymphdrüsenschwellungen constatirt wer-

I) $0 \mathrm{rth}$. 1. c. 763 .

$\left.{ }^{2}\right)$ In einem Falle (Eli Osakowski) auf unserer Abtheilung hatte die mikroskopische Untersuchung ein Sarcoma alveolare giganto-cellulare (angiosarcoma giganto-cellulare) aufgewiesen; in einem anderen Falle (Chaim Lindermann) waren die Knötchen keine rein spindelzelligen Sarcomen gewesen (vide Gazeta Lekarska 1889, Nr. 11, Aufsatz des Dr. Funk.)

3) Paltauf. Ueber lymphatisehe Neubild. d. Hant. II. Internat. Dermat. Congr. 1892. Bericht. Wien 1893, p. 147.

$\left.{ }^{4}\right) \mathrm{Ka}$ posi. Lehrb. pag. 885.

Arehiv f. Dermatol. u. Syphil. Band XIIX. 
den konnten. Richtig beobachtet auch Paltauf, dass "von Hautveränderungen bei Leukämie und Pseudoleukämie darf man erst sprechen, wenn es im Verlaufe des Allgemeinleidens zu solchen kommt". ${ }^{1}$ ) Kaposi ist der Meinung, dass zwischen diesen Formen jedoch gewisse Verwandtschaften und Uebergänge existiren können; er schlägt die Benennung "sarkoide Geschwïlste" vor und reihet an dieselbe ausser Sarcomatosis cutis auch Mycosis fungoides, Lymphodermia perniciosa nebst Leucaemia et Pseudoleucaemia cutis. Diese Benennung soll bezeichnen, dass genannte Geschwülste, obgleich nicht identisch mit den Sarcomen sensu strictiori - doch eine grosse Aehnlichkeit mit denselben haben. Max Joseph erklärt sich in seiner letzten Arbeit') mit der Aeusserung Kaposi's einverstanden in Betreff der Uebergangsformen zwischen den Symptomen dieser ganzen Gruppe. Die Benennung aber "sarcoide Geschwülste" würde er nur in seinem Falle von Sarcomatosis cutis und den vier Fällen Spieglers ${ }^{3}$ ) anwenden, denn diese entsprechen gar nicht den heute allgemein für den Begriff "Sarcoma" gestellten Forderungen; das typische melanotische Hautsarcom aber und das idiopathische multiple Pigmentsarcom der Haut (Typen Kaposi) entsprechen, der Aeusserung des Autors gemäss, vollkommen diesen Forderungen. Der Unterschied beruht auf folgendem: 1. Die Sarcome des Typus Kaposi sind Neubildungen im wahren Sinne des Wortes, dagegen sarcoide Geschwülste stellen sich nur als Infiltration der Cutisspalten mit fremden Zellen vor; 2. in den ersteren ist kein elastisches Fasernetz vorhanden, in den letzteren aber ist dasselbe gut färbbar und zeigt keine Veränderungen; 3. Mastzellen kommen in den ersteren, wie es Unna zeigte, nicht vor, dagegen in den letzteren sind diese Zellen, zwar in minderer Zahl, aber sicher vorhanden. Joseph gibt zu, dass die Entstehung dieser Tumoren vielleicht abhängig sei von einer chronischen Infectionskrankheit (Analogie mit Pseudo-

I) Paltauf l. c.

$\left.{ }^{2}\right)$ Max Josepì. Ueber Sarcomatose. Archiv f. Dermat. u. Syph. B. 46.1898 .

$\left.{ }^{3}\right)$ E. Spiegler. Ueber sogenannte Sarcomatosis cutis, Archiv f. Dermat. u. Syph. 1894. 
leukämie); Spiegler dagegen ist der Meinung, dass dieselben Uebergangsformen von Neubildungen zu chronischen Entzündungen (entzündliche Wucherung) sein können, ${ }^{1}$ )

Von hier $a b$ bleibt nur ein Schritt $z u$ den infectiösen Granulomen übrig, wenn auch gleich hinzuzufügen ist, dass der histologische Bau denselben nicht ganz entspricht ( $(\mathrm{n} \cap \mathrm{a})^{2}$ ) und dass ein Vorhandensein der Bakterien in diesen Tumoren bis heute, trotz vieler Nachforschungen, nicht nachgewiesen worden ist. Touton ${ }^{3}$ ) berichtet nur über einzellige Körperchen, welche den Sporozoen ähnlich waren.

Paltauf reihet in seiner vorzüglichen Arbeit "Ueber lymphatische Neubildung en der Haut" die ganze oben erwähnte Gruppe (Mycosis fungoides, Lymphodermia perniciosa Ka posi, Sarcomatosis cutis sowie Lymphadenia ossea Nothuagel) zu den sogenannten "Vegetationsstörungen " hinzu, indem er dieselben bezeichnet als „eine Störung, welche nicht in directer Abhängigkeit ron der Ursache steht, ihre Entwickelung nicht einer speciellen Ursache allein, sondern einer abnormen Reaction des Individuums, beruhend auf abnormer Vegetation, verdankt".4)

Zum Schluss möchte ich noch in dieser Hinsicht die Ansicht Semenow's ${ }^{5}$ ) anführen. Fr beobachtete zwei Fälle dieses Leidens mit dem Endergebniss der Section. Die Anamnese und die objective klinische Untersuchung, sowie auch ferner der Sectionsbefund und die mikroskopischen Untersuchungen ergaben Störungen und Veränderungen seitens des Nervensystems. Auf Grund seiner Untersuchungen kommt S. zu dem Ergebniss, dass es ein nervöses Leiden sei, durch paralytische Angioneurose bervorgerufen.

Wir ersehen daher, wie die Meinungsäusserungen verschieden sind in Anbetracht der Genese der in Rede stehenden Sarcome, sowie der Aetiologie dieses Leidens. - Was die

1) Spiegler. I. c. p. 173.

?) $0 \mathrm{rth}$. 1. c. p. 773 .

3) Touton. Arch. f. Dermat. u. Syph. 1893.

4) Paltauf. 1. c. p. 155 .

5) F. W. Semenow. Zur Pathol. d. Sarc. pigm. idiop. multipl. cutis Kaposi. Arch. d. Dermat. u. Syph. Band 36, 1896, Ref. pag. 430. 
Genese betrifft, so möchte ich jedoch bemerken, dass meiner Ansicht nach noch $\mathrm{zu}$ wenige Beobachtungen gemacht wurden in Anbetracht der ersten Bildungsphasen dieser idiopathischen multiplen Hautsarcome. Denn es ist klar, dass nur auf dem Wege der Untersuchungen von frühesten Entwickelungsphasen, wenn die zukünftige Geschwulst erst zu keimen beginnt, man zu etwaigen Resultaten in dieser Hinsicht gelangen könnte.

In einem von den zwei unten beschriebenen Fällen von idiopathischen multiplen Pigmentsarcomen der Haut (Typus Kaposi) wurden der mikroskopischen Untersuchung nicht nur schon ausgebildete Geschwïlste unterworten, sondern eben die Anfangsstadien ihrer Entwicklung. Ich glaube daher, dass dieselben eine detaillirte Beschreibung verdienen.

1. Fall. (Demonstrirt in der Warschauer ärztlichen Gesellschaft am 21./II. 1899.)

Mayer P., jüdischer Gemeindelehrer aus Bodżentyn (Gouv. Kielce, Polen), 52 Jahre alt, betrat die Abtheilung für Haut- und venerische Krankbeiten des Herrn Primarius Els en berg am israelitischen Krankenhanse zu Warsehau den 25. Januar 1899.

Stammt ans einer gesunden Familie, ist seit 20 Jahren verheiratet - Kinder gesund. Im siebenten Lebensjabre litt er an Caries des vierten Fingers am linken Fusse; im 30. Lebensjahre entwickelte sich bei ihm eine Augenkrankheit (Trachoma). Patient leidet an chronischem Erysipel localisirt in der Gegend des linken Kniegelenkes; dieselbe exacerbirt von Zeit zu Zeit.

Seine Krankheit begann mit dem Jahre 1891. Damals trat an der Radialseite des linken Handrückens Röthung und Verdickung der Haut in der Grösse eines Thalers auf; bald darauf bildeten sich kleine Knötchen auf dieser veränderten Hautpartie. Analoge Erscheinungen traten binnen kurzer Zeit auch auf der Haut des linken Fussrïckens, in der Gegend des linken Kniegelenks, ferner auf der oberen und unteren rechten Extremität auf. - In. letzterer Zeit ist der Kranke bedeutend abgemagert und fühlt sich schwach. - Patient ist von mehr als mittelmässiger Grösse, mit schwach entwickelter Musculatur und Fettpolster; Knochensystem normal. Die allgemeine Decke ist blass mit gelblichem Scheine; die für das Auge zugänglichen Schleimhäute sind blass übrigens normal.

Rechte obere Extremität. Die Hant des Handrückens ist gespannt, verdickt, dunkelbläulich -roth verfärbt; in derselben bemerkt man die Aussaat von ebenso verfärbten, ziemlich derben, das Hautniveau etwas überragenden Knötchen bìs zur Grösse einer kleinen Felderbse. Je ein solches Knötchen arch am ersten und fünften Finger sichtlich. Auf der Haut der'unteren Hälfte des Vorderarmes befinden sich Flecke von un- 
regelmässiger Gestalt, braungelber Farbe, ein wenig derber als die umgebende gesunde Haut selbst. Ebensolche Flecke kommen in der Ellenbogenregion und der hinteren Oberfläche der unteren Hälfte des Armes vor. Manche von den Flecken erheben sich etwas über das Hautaiveau - andere dagegen nicht; auf den Flecken sitzen in geringer Zahl kleine roth-braune Knötchen. Es sei bemerkt, dass auf den frischen Flecken die Knötchen nicht vorkommen; diese bilden sich erst mit der Zeit auf äIteren Fleoken.

Linke obere Extremitat. Auf dem Handrücken und in der Gegend des Handgelenkes finden wir Veränderungen analog der rechten Hand. Am Vorderarm, Ellenbogen und Arme sind die Flecke und Knötchen ebenso gebildet, wie die bereits oben geschilderten.

Rechte untere Extremität. Die Haut des rechten Fussrückens ist etwas angeschwollen, sehr verdickt, infiltrirt und festgespannt; diese Spannung ist besonders am Fussgelenke stark; die Farbe der Haut ist bläulich-roth. Am Unterschenkel ist die Haut ebenfalls etwas angeschwollen und dunkler als gewöhnlich tingirt. - Oberhalb des Malleolus int. befindet sich eine halbkugelige, bohnengrosse, weiche Erhöhung von schmatzigblauer Farbe mit flüssigem Inhalte; die Erhöbung schwindet auf Druck, um sich dann wieder zu zeigen, nachdem ein solcher nicht mehr ausgefubrt wird (degenerirtes Knötchen). Ein weniger nach oben sieht man ebenfalls eine solche Erhöhung, jedoch kleiner und nicht so weich. In der Kniegelenkregion auf der vorderen, inneren und äusseren Oberfäche bemerkt man Flecke in der Grösse einer Stengelbohne bis zur Dreimarkmünze etwas über das Hautniveau erhaben, gelb-roth bis röthlich-blau verfärbt, welche unter dem Drucke nicht verschwinden. - Die Flecke haben eine glatte Oberfä̈che; nur hier und da lassen sich kleine Knötchen fühlen.

Linke untere Extremität. Am Fussrücken und Fussgelenk kommen Hautveränderungen gleich der rechten Extremität vor. Am ersten, zweiten und dritten Finger findet man einige harte Knötchen von Hirsenkorn- bis Erbsengrösse von dunkelblauer oder blaurothunterlaufendex Farbe; ebensolche Knötchen, aber etwas grösser (bis zu einer Stengelbohne) befinden sich am inneren und äusseren Pande des Fusses und in der Fussgelenkgegend. - Die Haut des Unterschenkels ist etwas angeschwollen. In dem oberen dritten Theile des Untersebenkels, in der Kniegelenkregion, ferner in dem unteren vierten Theile des Schenkels auf ihrer hinteren sowie auch theilweise inneren Oberfäche befinden sich circa 20 ziernlich harte Knötchen von rosa oder violettrother Farbe bis zur Bohnengrōsse. Auf der Haut der vorderen Oberfläche des Kniegelenks findet man circa 15 viel grössere Knötchen, die beinahe die Grōsse einer Kirsche erreichen; dieselben sitzen auf breiter Basis, überragen hoch das Hautniveau; ibre Consistenz ist sehr derb, fast hart, die Farbe meistentheils violettroth-unterlaufen. Auf der äusseren Fläche der unteren Schenkelhälfte sieht man einen ziemlich grossen Fleck von un- 
regelmässiger Gestalt und gelb-rother Farbe; auf demselben bemerkt man erbsengrosse Knötchen.

In der recbten Glutaeusgegend befindet sich ein blau-rother unregelmässig gestalteter Fleck, ein wenig derber, als die ihm umgebende normale Haut; eben solche zwei Flecke, scharf begrenzt - in der linken Glutreusgegend - der eine in der Grösse eines Hühnereies, der andere etwas kleiner. In den tieferen Hautschichten lassen sich in der linken Glutaeusgegend ein und in der rechten zwei Knötchen fühlen, welche eine Stengelbohnengrösse erreichen; diese Knötchen überragen das Hautniveau nicht - nur erscheint die Haut über denselben etwas dunkler.

Die subcutanen Lymphdrüsen sind ausser den linken Leistendritisen nicht vergrössert. Innere Organe normal; es besteht eine leichte Arteriosclerosis. Die Zahl der rothen Blutkörperchen $=2,578.270$; das Verhältniss der weissen za den rotben $=1: 400$. Der Harn 1500 Ccm. pro die, klar, von saurer Reaction, sp. G. 1.021 ohne Eiweiss oder Zucker; es sind Phosphate in ziemlich grosser Anzahl vorhanden.

Das Nervensystem zeigt keine Abweichungen von der Norm.

o. d. Trachoma. O. s. Trachoma et Keratitis pannosa.

Während des Aufenthalts im Krankenhause hatte am 16./II. eine Exacerbation des Erysipels, an welchem Patient leidet und welche in der linken Kniegelenksgegend localisirt ist, stattgefunden; die localen entzündlichen Erscheinungen verschwanden nach vier Tagen.

Meiner Ansicht nach verdient dieser Fall aus diesem Grunde Berücksichtigung, weil er klinisch alle Entwicklungsphasen der Knötchen aufweist. - Es entstehen also zuerst die Flecke, welche anfänglich glatt erscheinen; im weiteren Verlaufe entwickeln sich in denselben kleine Knötchen, die nach und nach wachsen, beispielsweise bis zur Kirschengrösse (linkes Knie), Die zweite Absonderlichkeit dieses Falles ist der Process der Degeneration und Resorption der Knötchen, wie er am rechten Unterschenkel zu beobachten war. Dies weist darauf hin, dass die Knötchen geneigt sind, schon spontan zu verschwinden auf dem Wege der Resorption der degenerirten Masse des Tumors. Man darf also hoffen, dass bei einer guten Ernäbrung des Kranken und arsenikaler Behandlung (Arsenikales Serum von Prof. Nencki) eine völlige Resorption der Knötchen stattfinden kann. Es ist möglich, dass man diesen Fall zu jenen sehr seltenen Fällen ron multiplen idiopathischen Pigmentsarcomen der Haut zählen wird, bei welchen Alsenik sich wirksam erwiesen hatte. 
Sarcomata idiopathica multiplicia pigmentosa cutis (Kaposi). 215

Behufs histologischer Untersuchung wurden zwei ausgebildete Koötchen und auch zwei Stückchen Haut aus den Flecken excidirt. Das Untersuchungsergebniss war Folgendes:

1. Das Knötchen des rechten kleinen Fingers.

Das Knötchen hat makroskopisch die Grösse einer kleinen Felderbse und liegt in der pars reticuralis coriivon der Epidermis durch eine verhältnissmässig unveränderte Hautgewebschicht von 1-2 Mm. Stärke getrennt.

Der wichtigste Bestandtheil des Knötchens sind die Zellen. Wir treffen hier zwei Arten derselben an. Die einen sind spindelförmig, ihr Zellenkörper ist ziemlich lang aber schmal und färbt sich gut. Ihre Kerne, welche sehr stark die Farben aufsaugen, sind ebenfalls von spindelförmiger Gestalt und ziemlich grob granulirt. Solche Zellen sieht man im Knötchen am meisten; minderzählig ist die zweite Art repräsentirt. Dies sind grosse Zellen von ovaler, länglichovaler, seltener runder Gestalt mit sehr grossen blasenähnlichen Kernen, deren Form gewöhnlich der Gestalt des Zellenliörpers selbst entspricht. Ihre Kerne weisen ein zartes Netz auf, in dessen Maschen winzige Körnchen gelagert sind; sie besitzen auch 1-3 Kernkörperchen und färben sich viel schwächer, als die Kerne der Spindelzellen. Die Zellenkörper färben sich ebenfalls sehr schwach und sind deshalb blass, durchsichtig; in einen erscheint das Protoplasma homogen wie angeschwollen, in anderen wieder feinkörnig; in manchen wiederum sind kleine aber ziemlich viele Vacuolen bemerkbar, in Folge dessen der Zellenkörper einen netzartigen Bau darstellt. Die Spindelzellen, parallel gelagert, vereinigen sich in Bündel von unegaler Stärke; dieselben durchlaufen das Knötchen in verschiedener Richtung, weshalb man Längs-Quer- und Schrägschnitte vorfindet. Stellenweise bemerkt man, wie so ein Bündel sich gabelförmig theilt, oder seitwärts ein Zweigbündel abgeht; stellenweise wiederum kann man in der Mitte eines längs oder quer geschnittenen Bündels das mit Endothel ausgebettete Lumen eines Gefässes bemerken. - Was die Zellen der zweiten Art anbetrifft, so haben dieselben keine solch regelmässige Lage; sie sind in dem ganzen Knötchen zerstreut und bilden nirgends abgegrenzte Herde; nur hie und da um die Gefässe sowie auch in den 
Ecken der Gabelbündel sammeln dieselben sich in einer grösseren Anzahl an.

Den zweiten wesentlichen Theil bilden die Gefässe. Wenn man bei einer kleinen Vergrösserung das ganze Knötchen ins Auge fasst, so lassen sich breite Spalten bemerken, welche längs und quer durchlaufen; manche haben Seitenzweige. Das ganze Knötchen scheint durch diese Spalten wie auf einzelne lobi zertheilt zu sein. Die Spalten sind hie und da mit angequollenen, gewöhnlich aber mit platten Endothelzellen ausgebettet; stellenweise bemerkt man in den Spalten desquamirte Zellen, auch Fibrin und rothe Blutkörperchen, die sich hier wahrscheinlich aus den Hämorrhagien, die wir im Knötchen vorfinden, den Weg gebahnt haben.

Ausser den Spalten finden wir eine grosse Menge Capillaren vor. Es ist zu beachten, dass die Richtung dieser Gefässe gewöhnlich den spindelzelligen Bündeln entspricht; die Längsschnitte also der Gefässe stimmen mit denen der Bündel überein - gleichfalls wie auch die Querschnitte. In der Regel placiren sich diese Gefässe in der Mitte der Bündel, was bereits oben erwähnt worden ist. Stellenweise sieht man aber eine solch grosse Menge Capillaren, unmittelbar aneinander gelagert, durch feine Scheidewände auseinander getrennt, dass es den Eindruck eines kleinen Angioms macht. Die Capillaren sind meistentheils erweitert und mit rothen Blutkörperchen angefüllt; ihr Endothel ist angeschwellt, angequollen, mitunter in einem solch hohen Grade, dass es fast ganz das Lumen verschliesst. Die Gefässe sind gewöhnlich undeutlich von dem umgebenden Gewebe abgegrenzt; unmittelbar an ibren Wänden, als ob aus ihnen herauswachsend, liegen die Sarcomzellen.

In dem Knötchen sind wenige kleine Hämorrhagien vorhanden.

In der Uebergangszone sind Sarcomzellenzüge längs der Capillaren mit angeschwollenem Endothelium sichtbar; ferner sieht man, wie diese Zellen zwischen die Bündel des Bindegewebes eindringen und sie auseinander drängen; das Bindegewebe verschwindet allmälig und in dem Knötchen selbst ist es nicht mehr bemerkbar.

Die Färbung nach Tänzer-Unna hat nachgewiesen, dass das elastische Fasern im Knötchen nicht vorhanden 
ist. - Das Bindegewebe der Haut, ausserbalb des Knötcheus, ist normal geblieben; nur sind seine Bündel über dem äussersten Punkte des Knoten horizontal gelagert. Die grösseren Blutgefässe blieben unverändert; die Capillaren hie und da erweitert, mit dem wie oben veränderten Endothel, sind stellenweise von ebensolchen Zellen, wie sie in dem Knötchen rorkommen, umgeben. - Unweit des beschriebenen Knötchens befindet sich ein zweites sehr bleines, erst aufwachsendes. Auf Serienschnitten sieht man, wie neben zwei sehr erweiterten und mit rothen Blutkörperchen gefiullten Capillaren ein Herd entsteht, welcher sich aus eben solchen Zellen bildet, wie sie beim grösseren Knötchen geschildert worden sind. Anfänglich überwiegen an Anzahl die ovalen Zellen mit grossen schwach gefärbten Kernen; allmälig aber nehmen die spindelförmigen überhand. Der Charakter der Zellen selbst, ihre Gruppirung und ihr Verhältniss zu den Capillaren, deren nicht wenig in diesem Herde vorkommen, und die Veränderungen des Endothels - alles dies entspricht gänzlich den Bildern, die im Knötchen beschrieben worden sind. - Auch Hämorrhagien sind in diesem Herde vorhanden. Bei Durchsicht der Serienschnitte kann man die gesprengte Wandung eines von den zwei oben geschilderten Gefässen finden: aus dem Gefässe tritt Blut hervor, und die rothen Blutkörperchen dringen zwischen die Sarcomzellen.

Die Papillarschicht ist unverändert geblieben, nur hie und da trifft man in den Hautpapillen etwas erweiterte Capillaren ron ziemlich reichlichen lymphoiden Zellen umgeben an; uiber dem Knoten, dort, wo derselbe nahe der Epidermis liegt, sind die Papillen aussergewöhnlich niedrig und breit, stellenweise sind sie in Folge des Druckes sogar ganz verschwunden. Die Schweissdrüsen sind normal, nur um die Knäulchen herum ist das Hautgewebe mitunter zellenreicher. Die Talgdrüsen, arrectores pilorum, Haarscheiden und Haare sind nicht rerändert. Die Epidermis ausserhalb des Knötchens ist normal.

2. Ein Knötchen aus der Haut der linken Kniegelenksgegend von makroskopischer Grösse einer Stengelbohne.

Wenn ich mich auf detaillirte Beschreibung dieses Präparates einlassen wollte, so wäre ich gezwungen, Wort für Wort 
zu wiederholen, was ich beim ersten Knötchen bereits erläutert habe, denn sein histologischer Bau ist vollkommen identisch: also dieselbe Lagerung in dem Aautgewebe, derselbe Reichthum an Gefässen, zwei Typen von Zellen und ihr Verhältniss zu den Gefässen etc. Wenn ich dennoch von diesem Knötchen erwähne, so ist es in erster Linie nur deshalb, um festzustellen, dass der Bau der Knötchen in verschiedener Grösse, also auch im verschiedenen Stufenalter, eben ganz gleich ist; und zweitens - um ein Bild aus dem Präparate zu schildern, wo sich das Verhältniss der Spindelzellen zu den Gefässen aussergewöhnlich hervorhebt, - namentlich ihre Herkunft von dem Perithel; ferner möchte ich noch die Aufmerksamkeit auf manche Eigenthümlichkeiten bezüglich dieses Knötchens leiten wollen.

Es stellt sich also folgendes Bild dar: Im Gesichtsfelde sieht man die Lumina ron fünf Gefässen - drei sind quer geschnitten, zwei längs und eins schräg. Die Gefässe sind erweitert; ihre Wandnng besteht aus einer Schicht flacher, etwas angequollener Zellen mit angeschwollenen Kernen. Diese Gefässe sind nicht abgegrenzt von dem umgebenden Gewebe des Tumors, ja dies selbst scheint für sie eine enorm dicke Wandung zu bilden. Dieses Geschwulstgewebe ist aus den schon oben geschilderten zwei Zellentypen geschaffen und bildet also für die Gefässe Scheidewände; eine von dieser Scheidewand ist nur aus Spindelzellen zusammengesetzt. Man sieht überhaupt, dass rings um das Endothel dieser kleinen Gefässe einige Schichten grosser spindelförmiger Zellen parallel ihrer Wand gelagert sind; unter einem spitzen oder rechten Winkel ron diesen Gefässen zweigen kleine Capillaren $a b$, ebenfalls von einigen Schichten spindelförmiger Zellen umgeben: letztere liegen nun senkrecht oder schräg gegenüber jenen Zellenschichten, welche ihr Muttergefäss umringen. Hier tritt also deutlich die Entstehung der spindelförmigen Zellen von den Perithelien der Gefässe auf.

In dem unteren Theile des Knötchens sieht man zwei längsgeschnittene Arrectores pilorum mit gut sich färbenden Kernen. Die zwei Muskeln bilden einen Winkel; ihre vereinigten Enden verschwinden in dem das Knötchen um- 
gebenden Hautgewebe, dagegen die auseinander gehenden Enden dringen in den Tumor selbst hinein. Man empfindet also don Eindruck, als ob das Knötchen auf den Muskeln wie eine Frucht auf der Stengelspitze sässe. Im Knötchen sieht man auch von unten eindringende Bindegewebsstränge; in ihren Spalten bemerkt man hie und da Sarcom-oder Wanderzellen.

Ausser den gewöhnlichen zwei Typen finden wir in diesem Knötchen noch Wanderzellen vor. Ich möchte ihre Anwesenheit dem chronischen Erysipel zuschreiben, an welchem Patient leidet und welches sich eben in der linken Kniegelenkgegend localisirt (vide die Krankheitsgeschichte).

Während des Aufenthalts des Kranken im Hospital hatte eine Exacerbation des Erysipels stattgefunden; nach dem Verschwinden der localen Entzündungserscheinungen wurde 10 Tage darauf das Knötchen excidirt.

In diesem Knötchen findet man reichlicher Hämorrhagien vor, als im vorigen und in den cylindrischen Zellen der Malpighischen Schicht körniges braunrothes Pigment.

3. Ein Stückchen Haut aus einem Fleckchen ron hellrother Farbe excidirt. (Rechter Arm.) In diesen Präparaten findet man die hauptsächlichsten pathologischen Erscheinungen in den Gefässen und rings um dieselben. Der pathologische Process nimmt seinen Ursprung aus den Gefässen, welche um die Haarscheiden, Talgdrüsen und Haarmuskeln gelegen sind. Die hier durchziehenden Capillaren haben gewöhnlich ein Endothel mit sehr angequollenen Kernen, die Zellen selbst erscheinen höher und dicker, was besonders gut zu sehen ist an quergeschnittenen Gefässen; letztere sind mitunter etwas erweitert, andere wiederum nicht erweitert. In den Gefässen kleineren Massstales verschliesst mitunter das veränderte angequollene Endothel gänzlich das Lumen, oder verengt es so sehr, dass sogar ein rothes Blutkörperchen darin nicht Platz finden könnte. Rings um die so veränderten Gefässe findet man Streifen oder Herde der Zellen, welche den zwei im Knötchen geschilderten Zellentypen angehören - es sind dies also wiederum Spindelzellen und ovale Zellen mit grossen, schwach sich färbenden Kernen. Diese Zellen befinden sich direct an der Endotbelwand der Gefässe, mit welcher sie ein 
untrennbares Ganzes bilden, als ob sie von da herauswachsen möchten. Stellenweise umringen diese Zellen die Gefässe, eine Scheide bildend; gewöhnlich aber ist die Wucherung der Zellen an einer Seite des Gefässes grösser, als an der anderen, und dadurch präsentirt sich unseren Augen ein Buckel aus der Wand des Gefässes herauswachsend, oder auch ein breiter Streifen, der an einer Seite haftet. In einem solchen Streifen kann man manchmal eine gewisse Regelmässigkeit der Gruppirung der Zellen vorfinden, nämlich: Zwischen zwei spindelzelligen Schichten liegt eine solche aus ovalen Zellen gebildete. Diese beiden spindelzelligen Schichten sind nichts anders als zwei Gefässe mit veräıdertem Endothel und von wucherndem Perithel umgeben.

Da solche Veränderungen, wie oben bereits erwähnt wưrde, in jenen Gefässen, welche um die Haarscheiden, Talgdrüsen und $\mathrm{m}$. arrectores pilorum liegen, beobachtet werden, so sind genannte Scheiden, Drüsen und Muskeln mitunter gänzlich von Geschwulstzellen umringt. Jedoch weder die Elemente der Haarscheiden, noch der Talgdrüsen nehmen an der Tumorbildung einen activen Antheil. Was aber mit den Hautmuskeln geschieht, das kann man aus den ausgebildeten Knötchen nicht ermitteln, denn genannte Muskeln sind dort gar nicht vorhanden und wenn sie mitunter auch vorkommen - dann nur auf der Peripherie der Knötchen und dazu wenig verändert. Auf den Präparaten aber, von welchen jetzt die Rede ist, sieht man, dass die arrectores pilorum von Sarcomzellen umgeben, evident einem regressiven Processe unterliegen. Man sieht z. B. Folgendes: Ein längsgeschnittener arrector pili liegt im Sarcomgewebe, dessen einzelne Zellen auch zwischen seine. Fasern dringen. Die Kerne der Muskelfasern sind schwach, kaum sichtlich gefärbt; die Fasern selbst sind wie auseinandergerückt, feinkörnig und färben sich mittelst Eosin sehr schwach; hin und wieder sieht man in denselben auch kleine Vacuolen. Ohne Zweifel ist das ein regressirer Process, umsomehr, da andere Muskeln auf diesem Präparate, welche nicht von Sarcomzellen umringt sind, sich gut färben und keine Veränderungen aufweisen. Wahrscheinlich, indem das. Knötchen wächst, degeneriren die Muskeln und verschwinden allmälig. Auf 
diesem selben Wege gelangten sie gewiss auch in den ausgebildeten Knötchen zum Verschwinden, und ihre Stelle nehmen nun die Sarcomzellen ein.

Aber die geschilderten Gefässveränderungen kommen nicht nur um die Haarscheiden, Talgdrüsen und Hautmuskeln vor, sondern dieselben sind auch in dem ganzen Hautgewebe zerstreut, wiederum Zellenstreifen oder Zellenherde bildend. Und nicht nur an den Capillaren, sondern auch rings um die kleinen Arterien sieht man solche Herde; die Arterien aber selbșt haben ein angeschwollenes Endothel und ihre media, von dem umgebenden Gewebe undeutlich abgegrenzt, ist mit Sarcomzellen infiltrirt.

Die Hautpapillen sind unrerändert geblieben; Schweissdrüsen und das Unterhautgewebe sind normal. In den cylindrischen Zellen der Malpighischen Schicht findet man ziemlich viel körniges braunrothes Pigment vor; dagegen in dem Hautgewebe selbst sind weder Hämorrhagien noch Pigment vorhanden.

4. Ein Stückchen Haut aus einem Flecke am rechten Arm. - Das Fleckchen war von dunklerer Farbe und von etwas derberer Consistenz, als das vorherige, unter Nr. 3 beschriebene.

Die pathologischen Yeränderungen, welche man auf diesen Präparaten vorfinden kann, bestehen in kleinzelliger Infiltration, welche herdförmig in dem ganzen Hautgewebe zerstreut ist. Um die etwas erweiterten, aber sonst unveränderten Blutgefässe herum befinden sich Herde, die aus Wanderzellen bestehen. Diese Zellen dringen auch zwischen die Bündel des umgebenden Hautgewebes ein; in Folge dessen sieht man in der Nachbarschaft der Gefässe stellenweise eine Art Netz, dessen Fäden aus Wanderzellen gebildet sind. In den so entstandenen Maschen liegen Bindegewebsbündel, manchmal etwas dünner und schwächer als gewöhnlich gefärbt. Solche Herde sind im ganzen Corium zerstreut, aber ihr grösster Theil befindet sich in den oberen Schichten desselben.

Talg- und Schweissdrüsen, Haarscheiden und Haarmuskel sind normal. In den cylindrischen Zellen des stratum Malpihgii ist braunrothes Pigment vorhanden; sonst ist die Epidermis unverändert geblieben. 
Alle oben beschriebenen Präparate (die Hautstückchen aus den Flecken und die Knötchen) wurden nach Gram, Gram-Kühne auch mit Eosin-Methylenblau auf Bakterien untersucht, aber immer mit einem negativen Ergebnisse.

II. Fall. Zum Beschreiben dieses Falles dienten mir die schon angefertigten und mir freundlichst überlassenen Präparate meines hochverehrten Lehrers, des Herrn Dr. med. A. Els enberg.

Der Kranke und die Präparate wurden demonstrirt in der ärzt. lichen Warschauer Gesellschaft am 1./III. 1898.

Abrabam Z, 50 Jahre alt, betrat unsere Abtheilung am 26. Januar 1898. - Die ersten Krankheitserscheinungen bemerkte er im Jabre 1894; in dieser Zeit zeigte sich gleichzeitig auf dem rechten und linken Fussrủcken eine stablblaue Verfärbung der Haut, die auch etwas dicker warde. Das Gehen war nicht erschwert, auch fühlte er keine Schmerzen. Diese Erscheinungen existirten zwei Jahre ohne jegliche Veränderung; nachber aber begann sich die Haut an den Fingern der Füsse gelblich $\mathrm{zu}$ verfärben und später überging diese gelbe Farbe in eine ebenfalls stahlblave. Beim Gehen fühlte er Schmerzen und hauptsảchlich unter dem rechten Halux. Er wandte sich an Aerzte: es wurden eine Mercurialbehandlung (36 Einreibungen) und Schwefelbäder verordnet.

Beim Fintreten in das Hospital war der Status praesens folgender:

Patient von mittelmässiger Grösse mit schwach ontwickelter Musculatur. und Fettpolster; das Knochenskelet normal.

Die Haut an den Fingern des rechten Fusses, hauptsächlich auf ihren dorsalen Oberflächen, ist von diffuser stahlblaner Farbe; die Finger verdickt und ungestaltet; die Haut ist dick, infiltrirt und sehr derb. Auf den plantaren Oberfächen mancher Finger, hauptsächlich des Haiux, findet man erbsengrosse Knötchen, von hellrother Farbe, ziemlich derber Consistenz und sehr schmerzhaft.

Die stahlblaue Verfärbung der Haut an den Fingern verbreitet sich unmittelbar auf den Fussrücken, aber hier ist sie schon viel heller; nur die Seiten des Fusses sind von tiefstablblauer Farbe. Die Haut, welche sehr verdickt ist, so dass ihre Stärke bis $1 \mathrm{Cm}$. beträgt und aussergewöhnlich infiltrirt ist, zeigt sich derb und stark gespannt. Die Haut lässt sich auf dem Fussrücken nicht falten. Auf der so veränderten Haut sieht man ziemlich niedrige, platte Erhöhungen und Knötchen in der Grōsse eines Nadelkopfes bis zur Stengelbohne; dieselben sind derb, von dunkelblauer Farbe und schmerzhaft. Die Haut anf der Ferse ist von rotber Farbe, verdickt und sehr derb; das Unterhantgewebe ist angeschwollen. Die Ferse ist sehr schmerzhaft, so dass das Gehen unmöglich ist. - Die Hantveränderungen endigen scharf in der Höhe des Fussgelenkes. Analoge Veränderungen, aber in einem schwächeren Grade, finden wir auf der Haut des linken Fusses; hier sind dieselben nicht so deutlich und vorgerückt, als auf dem rechten F'usse, obwohl sie gleichzeitig entstanden sind. 
An anderen Körpertheilen ist die allgemeine Decke ohne Veränderung. Die subcatanen Lymphdrüsen sind nicht vergrössert. Die für das Auge zugänglichen Schleimhäute sind ziemlich blass. Die Schleimhaut des weichen Gaumens ist dunkelrotb; diese Verfärbung bricht an der rechten Seite, an der Grenze des harten Gaumens, deutlich ab - an der linken aber steigt sie nicht so boch. Auf diesem dunkelrothen Grunde sieht man hie und da kleine rosablasse Fleckchen. Die Uvula ist ebenfalls roth, aber blässer, als der weiche Gaumen. Bei der Palpation stellt es sich heraus, dass der weiche Gaumen etwas derber als gewöhnlich ist, obwoll das Auge keine Infiltration entdecken kann; beim zarten Befühlen mittelst dem Finger constatirt man in dem weichen Gaumen kleine, flache, begrenzte Infiltration-Knötchen. Das Bewegungsvermögen des Gaumens ist normal. Die Schleimhaut der hinteren Wand der Pharynx ist etwas dicker und von etwas dunklerer Farbe, als gewöhnlich. Am Arcus palato-pharyngeus dexter befindet sich ein Knötchen von der Grösse eines kleinen Nadelkopfes, welches GranulationsWucherungen ähnlich ist. Auf der Schleimbaut des harten Gaumens befinden sich an der rechten Seite zwe: Fleckchen von unregelmässiger Gestalt und dunkelrother Farbe; die Palpation zeigt, dass es Infiltrate sind. An der linken Seite sieht man ein ebensolches Fleckchen.

Die inneren Organe sind ohne Veränderungen, nur stehen die Lungengrenzen etwas niedriger, sowie auch die untere Grenze der Leber.

Der Harn $1500 \mathrm{Ccm}$. p. die ist kiar, von saurer Reaction, sp. Gewicht 1.020 ohne Zucker noch Eiweiss.

Behufs mikroskopischer Untersuchung wurde ein Stückchen Haut aus dem linken Fussrücken, sowie auch ein Knötchen aus dem harten Gaumen tief excidirt.

Der histologische Bau dieser excidirten Stückchen ist vollkommen mit der Structur der Knötchen des ersten Falles identisch, es wäre also unnöthig, denselben nochmals ausführlich zu wiederholen. Ich möchte nur wieder die Aufmerksamkeit dahin leiten, dass die Gefässe, welche auch in diesen Gebilden in grosser Anzahl vorkommen, nirgends eigene Wände besitzen; eigentlich bildet ihre Wandung das sie umgebende sarcomatöse Gewebe. Auch hier liegen die Gefässe in der Mitte der spindelzelligen Bündel, deren Zellen von den Perithelien entstanden sind.

Ferner möchte ich noch auf folgende Eigenthümlichkeiten hinweisen.

Die Haut des Fussrückens wurde aus einer knötchenlosen glatten Stelle excidirt, doch war sie sehr verdickt. Die hier bestehenden Veränderungen gehören höchstwabrscheinlich zu 
schon lang dauernden, denn dieselben beschränken sich nicht bloss auf das pars reticularis corii, sondern sie greifen von einer. Seite auf die Papillarschicht, welche sie allmälig vernichten, von der anderen Seite auch auf das subcutane Gewebe über. Hier dringen die Sarcomzellen zwischen die Fettzellen, Streife oder Netze bildend; die Fettzellen aber selbst verschwinden allmälig. Auf diesen Präparaten kann man auch die Contouren der Knäuel und der Ausführungsgänge der Schweissdrüsen bemerken. Manche von denselben sind noch ziemlich deutlich abgegrenzt ron dem sie umgebenden Sarcomgewebe, andere dagegen sind mit ihm schon gänzlich verschmolzen. Ausserdem findet man sehr reichlich Hämorrhagien und viel Pigment vor.

Man muss also diese Veränderungen als eine diffuse sarcomatöse Degeneration der Haut des Fusses betrachten.

Was das Knötchen aus der Schleimhant des harten Gaumens anbetrifft, so ist zu erwähnen, dass dasselbe in dem Gewebe der Schleimhaut liegt; die Papillen aber und das Unterschleimhautgewebe nebst den Drüsen sind normal. Das Epithel ist unverändert. Im Knötchen findet man auch Hämorrhagien und Pigment.

Dieser Fall ist aus dem Grunde bemerkenswerth, dass die Knötchen in der Schleimhaut des harten und weichen Gaumens localisirt waren, was jedenfalls zu keinen häufigen Erscheinungen gezählt werden kann. Holländer demonstrirte in der Berliner ärztlichen Gesellschaft einen analogen Fall, wo die Knötchen in Kirschkerngrösse, auch kleiner, sich auf der Schleimhaut des harten Gaumens, der Zunge, am Augenlidrande und auf der Wange sich bildeten; doch erwiesen sich mikroskopisch dieselben als pigmentirte Lymphosarcome. ${ }^{1}$ )

Aus dem oben geschilderten histologischen Knötchenbau und besonders aus der Beschreibung ihrer ersten Entstehungsphasen ergibt sich direct die anatomische Diagnose: Angio-sarcoma fusocellulare peritheliale cutis.

In der That, jene Streifen und Buckel, welche in den ersten Entwickelungsphasen längs und ringsherum an beschränkten

1) Berliner klin. Wochenschr. 1898. Nr. 5. 
Stellen der Gefässe zu sehen sind, entstehen infolge Wucherung der Perithelien; denn in den frühesten Stadien bemerken wir zuvörderst die Schwellung der perithelialen Zellen und nachher ihre Wucherung, infolge dessen sich die obengenannten, Gefässe umgebenden Scheiden bilden. Diese Scheiden gleichen jenen spindelzelligen Bündeln, welche in den schon ausgebildeten Knötchen in verschiedener Richtung laufen, sich gabelförmig theilen und Seitenzweige aufweisen; in der Mitte aber der Bündel sieltt man das Lumen dieses Gefässes, aus dessen Perithelium das Bündel selbst entstanden ist, und dessen Wandung nun das infolge perithelialer Wucherung entstandene sarcomatöse Gewebe bildet.

Wie entsteht also das Knötchen? Diese Frage möchte ich folgendermassen beantworten. Das gleichzeitige und dazu mitunter acute Auftreten zahlreicher Knötchen, das zeitweise Verschwinden älterer und das parallele Entstehen von frischen Knötchen erinnert jedenfalls an eine chronische Infectionskrankheit. Ls ist möglich, dass die in den Kreislauf des Blutes eindringenden Bakterien vor allem ihren Haltepunkt an den rom Herzen entferntesten Stellen suchen; zu diesen gehören vor allem die Hände und hauptsächlich die Füsse, wo die Circulationsbedingungen gewöhnlich herabgesetzt sind. Hier entsteht also, infolge Irritation eine Erweiterung der Blutgefässe und Emigration der weissen Blutkörperchen. Wir haben also ein Bild, das dem unter Nr. 4 (erster Fall) geschilderten entspricht. Die Wanderzellen verschwinden nachher, wahrscheinlich in den Blutkreis zurückkehrend, auf dem Wege der Lymphspalten und Lymphgefässe; dagegen beginnt, infolge derselben Irritation die Wucherung des Perithels der Blutgefässe. Dam entstehen eben Streifen und Herde um die Gefässe herum (Nr. 3 des I. Falles), die in den Anfangsstadien constant un die Haarscheiden, Talgdrüsen und arrectores pilorum gelagert sind. Im weiteren Verlaufe entstehen die grösseren Knötchen infolge centralen Wachsthums und Verschmelzung einzelner Herde miteinander.

Die spindelförnigen Zellen muss man also als von dem Perithelium entstanden betrachten. Was num aber den zweiten Typus der oben beschriebenen längsovalen Zellen mit grossen Kernen anbetrift, so erinnern dieselben sehr an diejenigen, 
welche ron A. Elsenberg in den Sarcomen des Xeroderma pigmentosum beschrieben wurden. ${ }^{1}$ )

Höchstwahrscheinlich sind es dieselben spindelförmigen, also von den Perithelien entstandenen Zellen, nur modificirt und degenerirt infolge Aufnahme von Bakterien. Denn einerseits kann man keine andere Quelle ihrer Herkunft entdecken, anderseits aber weisen darauf jene regressiven Veränderungen, welche man in diesen Zellen vorfinden kann, namentlich: die schwache Färbung ihrer blasenförmigen Kerne, die schwache Färbung der Zellenkörper, welche mitunter durchsichtig, feinkörnig oder angequollen erscheinen, oder auch Vacuolen enthalten. Eine solche Auffassung würde auch in Uebereinstimmung sein mit der bereits ausgesprochenen Meinung über die infectiöse Natur des in Rede stehenden Leidens.

Ich möchte auch noch die Aufmerksamkeit auf den Umstand leiten, dass die Hämorrhagien wahrscheinlich zu dem Anfangsstadium dieses pathologischen Processes nicht gehören; - demnach also können sie denselben auch nicht verursachen; in meinem Falle wenigstens wurden keine Hämorrhagien in den ersten Entwickelungsphasen dieser Sarcome vorgefunden. Dagegen treten Hämorrhagien in den ausgebildeten Knötchen constant auf und sind dieselben um so reichlicher vorhanden, je grösser und älter das Knötchen ist. In solchen Knötchen eben gibt es auch immer viel Pigment. Die Hämorrhagien hängen gewiss rom Zersprengen der schwachen Wände neugebildeter Gefässe $a b$, welche wahrscheinlich mit dem Wachsthum des Knötchens entstehen, ${ }^{2}$ ) oder auch werden sie durch das Zerspringen der degenerirten Wandungen der präexistirenden Gefässe bedingt.

1) Ant. Elsenberg. Xerodema pigmentosum (Kaposi), Melanosis lenticularis progressiva (Pick), Arch. f. Dermat. u. Syph. 1890, pag. 49.

2) Vide auch Perrin 1. c. p. 58-59. 